\title{
The Galactic Halo in Hydrostatic Equilibrium
}

\author{
P.M.W. Kalberla, J. Pietz, and J. Kerp \\ Radioastronomisches Institut der Universität Bonn, Auf dem Hügel 71, 53121 Bonn, \\ Germany
}

\begin{abstract}
The large scale distribution of gas, magnetic field and cosmic rays in the Galaxy is explored. We analyze recent all-sky surveys of $\mathrm{H}$ I gas (Leiden/Dwingeloo survey), soft X-ray radiation (ROSAT) and high energy gamma-rays (EGRET> $100 \mathrm{MeV}$ ) in combination with the $408 \mathrm{MHz}$ survey. From these observations we derive a Galactic halo model consistent with hydrostatic equilibrium conditions.

The large scale equilibrium configuration consists of two separate domains with different properties and scale sizes:

1) The gaseous halo consists of collisionally ionized gas at $T=10^{6.2} \mathrm{~K}$ as well as $\mathrm{HI}$ and has an exponential scale height $h_{z} \sim 4.4 \mathrm{kpc}$. The radial distribution is characterized by the galactocentric scale length $A_{1} \sim 15 \mathrm{kpc}$. All components of the halo - gas, magnetic field and cosmic rays - contribute equally to the pressure. The magnetic field in the halo is oriented parallel to the Galactic plane.

2) The disk extends up to a scale height of $h_{z} \sim 1 \mathrm{kpc}$ and is associated with irregular magnetic fields. Here the pressure of the magnetic field is only $1 / 3$ of the gas pressure. The cosmic rays are only weakly coupled to the disk.
\end{abstract}

\section{Model Assumptions}

We assume that the average properties of the Galactic halo can be described by a hydrostatic equilibrium situation as defined by Parker (1966) and more recently by Boulares \& $\operatorname{Cox}(1990, \mathrm{~B} \& \mathrm{C})$. We represent the gaseous disk-halo system by three basic components with their characteristic vertical exponential scale heights $h_{z}$ : the neutral interstellar medium (ISM) with $h_{z}=400 \mathrm{pc}$ as used by B\&C, the diffuse ionized gas (DIG) with $h_{z}=1 \mathrm{kpc}$ as described by Reynolds (1997), and a halo gas with $h_{z}=4.4 \mathrm{kpc}$.

New in our hydrostatic model are the parameters for the gaseous halo which are from Pietz et al. and Kalberla et al. (both, this volume). The halo is composed of an X-ray emitting gas of a temperature of $T=10^{6.2} \mathrm{~K}$ and a highly turbulent neutral component. Both halo components, according to their best-fit distributions, have similar density distributions and an identical scale height of $h_{z}=4.4 \mathrm{kpc}$.

Fig. 1 displays the vertical density distributions of the gas phases (ISM, DIG and halo gas) in the solar vicinity. Fig. 2 displays our normalized radial density distribution $g_{1}$ at $z=0 \mathrm{kpc}$ which was selected according to Taylor $\&$ Cordes (1993) after modifying the radial scale length to $A_{1}=15 \mathrm{kpc}$. 


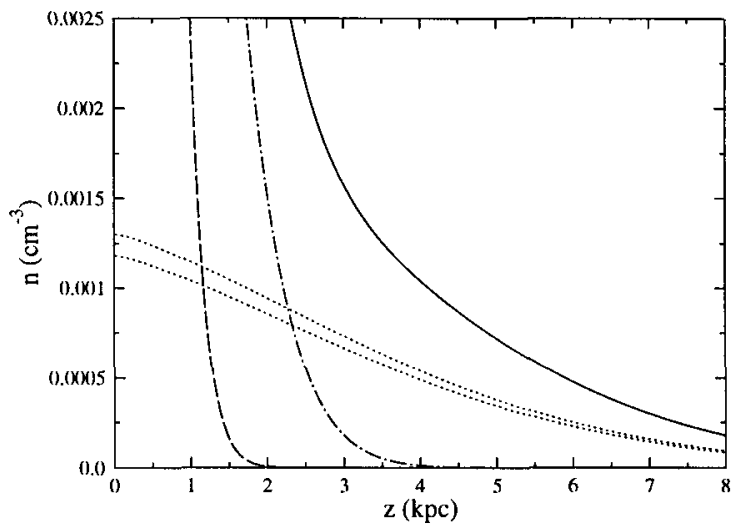

Fig. 1. Vertical density distribution of the gas components in the solar vicinity. The dotted lines mark the halo gas (lower: neutral gas, upper: ionized gas). The neutral ISM gas is indicated by the dashed line. The Galactic DIG layer is represented by the dot-dash line; the sum of all components is given by the solid line.

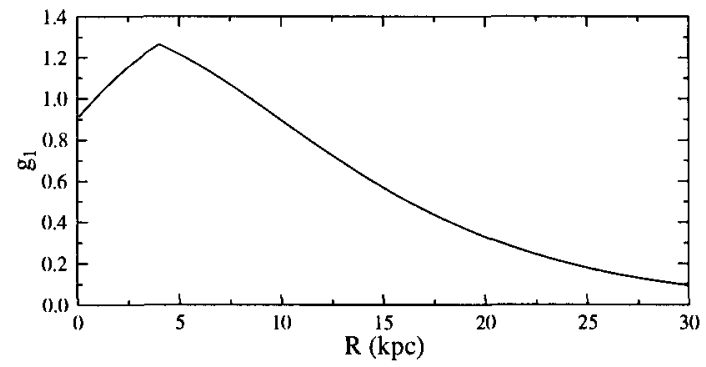

Fig. 2. Radial distribution for the gas density at $z=0 \mathrm{kpc}$ normalized to the solar vicinity, $g_{1}\left(R_{\odot}\right)=1$. The scale length is $A_{1}=15 \mathrm{kpc}$.

\section{Gas, Magnetic Field and Cosmic Rays in Equilibrium}

Following Parker's (1966) suggestion we studied whether gas, magnetic fields and cosmic rays in the Galactic halo may be in pressure equilibrium. Indeed, model calculations fit best the all-sky-averaged observations if one assumes such an equilibrium situation. To verify Parker's suggestion we have modelled the synchrotron emission at $408 \mathrm{MHz}$ as observed by Haslam et al. (1982) as well as the $\gamma$-ray emission as observed with EGRET at energies $>100$ $\mathrm{MeV}$ (Fichtel et al. 1994). A detailed description of the model calculations and a comparison with the observations are beyond the scope of this short contribution; details will be published elsewhere.

We derive a complete pressure equilibrium between gas, magnetic field and cosmic rays for the halo component. In the halo, the magnetic field is oriented parallel to the Galactic plane. Close to the disk $(|z|<1 \mathrm{kpc})$ the 
pressure of the magnetic field and the cosmic ray component is only $1 / 3$ of the gas pressure. The orientation of the field is irregular.

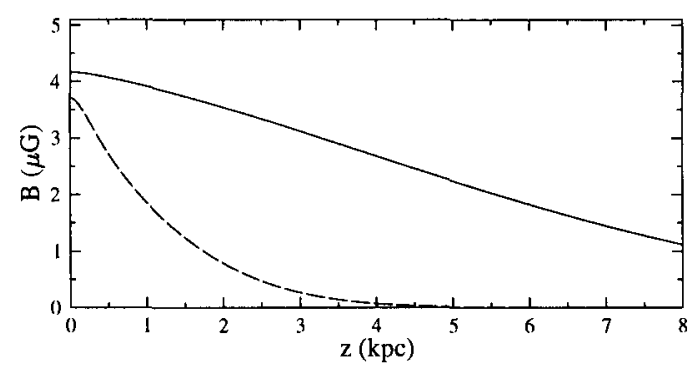

Fig. 3. Vertical distribution of the large scale magnetic-field components as expected from our model for the solar vicinity $\left(R=R_{\odot}\right)$. The solid line represents the regular magnetic field component in the halo which is oriented parallel to the Galactic plane. The irregular component of the magnetic field which is associated with the disk is marked by the dashed line.

The strengths of regular and irregular magnetic field components derived by us as a function of vertical distance from the Galactic plane are plotted in Fig. 3. We can trace the $\gamma$-ray emission up to intermediate latitudes with good agreement between model and observations. In Fig. 4 we display the derived average radial distribution of the $\gamma$-ray emissivity for $z=0 \mathrm{kpc}$. Our radial distribution is in good agreement with the one derived by Webber et al. (1992) which is plotted for comparison. We note further that our vertical scale height is also consistent with Webber et al. (1992).

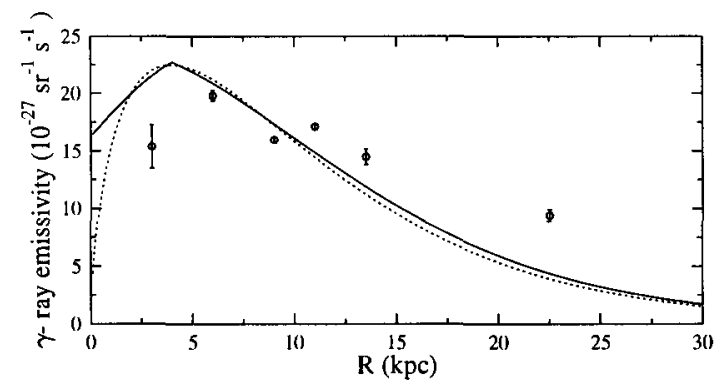

Fig. 4. Radial distribution of the $\gamma$-ray emissivity derived from our model (solid line). The dashed line represents the best fit model given by Webber et al. (1992, Fig. 2). The data are obtained from a gradient model analysis of EGRET diffuse Galactic $\gamma$-ray emission (Strong \& Mattox 1995). 


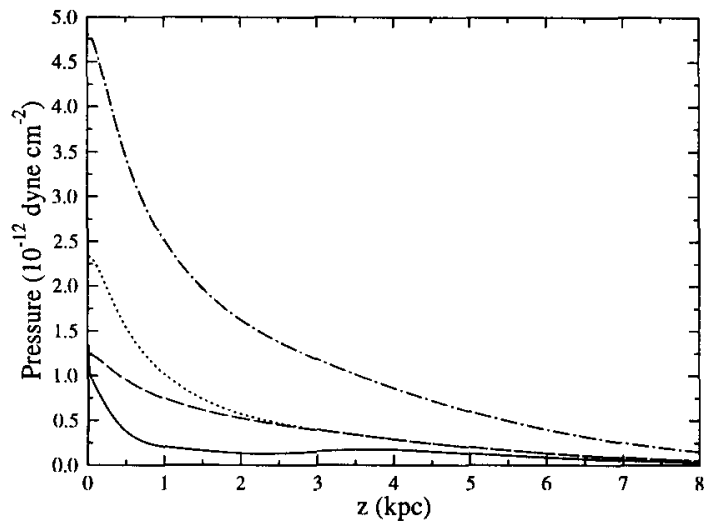

Fig. 5. Vertical pressure distribution in the solar vicinity for gas (dotted), magnetic field and cosmic rays (dashed). The solid line represents the minimum gas pressure required for stability. The dash-dotted line gives the total pressure. Stability for the disk-halo system is ensured for $|z| \lesssim 4.5 \mathrm{kpc}$.

Since Parker's (1966) classical publication it was not possible to describe a halo which is in quantitative agreement with observations and which is stable at the same time. Applying stability criteria as derived by LachièzeRey et al. (1980, see B\&C for discussion) we find that our modelled disk-halo system is stable. In Fig. 5 we display our vertical pressure distributions for gas, magnetic fields and cosmic rays. Stability is ensured if the gas pressure exceeds a minimum pressure given by the lower curve. Instabilities may be caused by small scale pressure fluctuations. However, according to Fig. 5 the formation of high velocity clouds due to such fluctuations appears to be restricted to $z \gtrsim 4.5 \mathrm{kpc}$.

Remarkable is the high gas pressure which results from our model. In the local vicinity we expect $\mathrm{nT} / k \sim 17000 \mathrm{~K} \mathrm{~cm}^{-3}$.

\section{References}

Boulares A. \& Cox D.P., (1990): ApJ 365, 544 (B\&C).

Fichtel C.E. et al. (1994): ApJS 94, 551.

Haslam C.G., Stoffel H., Salter C.J. \& Wilson W.E., (1982): A\&AS 47, 1.

Lachiz̀e-Rey M., Asséo E., Cesarsky C.J. \& Pellat R., (1980): ApJ 238, 175.

Parker E.N., (1966): ApJ 145, 811.

Reynolds R.J. (1997): in Proc. of 156. WE-Heraeus-Seminar on 'The Physics of

Galactic Halos', eds. H. Lesch, R.-J. Dettmar, U. Mebold, \& R. Schlickeiser, Akademie Verlag, Berlin, 57.

Taylor J.H. \& Cordes J.M., (1993): ApJ 411, 674.

Strong A.W. \& Mattox J.R., (1995): A\&A 308, L21.

Webber W.R., Lee M.A. \& Gupta, M. (1992): ApJ 390, 96. 